\title{
Mevastatin promotes healing by targeting caveolin-1 to restore EGFR signaling
}

\author{
Andrew P. Sawaya, ${ }^{1,2}$ Ivan Jozic,, Rivka C. Stone, ${ }^{1}$ Irena Pastar, ${ }^{1}$ Andjela N. Egger, ${ }^{1}$ \\ Olivera Stojadinovic, ${ }^{1,3}$ George D. Glinos, ${ }^{1}$ Robert S. Kirsner, ${ }^{1,4}$ and Marjana Tomic-Canic ${ }^{1,2}$ \\ 'Wound Healing and Regenerative Medicine Research Program, Dr. Phillip Frost Department of Dermatology and \\ Cutaneous Surgery, and ${ }^{2}$ Molecular and Cellular Pharmacology Graduate Program in Biomedical Sciences, University of \\ Miami Miller School of Medicine, Miami, Florida, USA. ${ }^{3}$ Immunology, Infection and Inflammation Graduate Program, \\ Faculty of Medical Sciences, University of Kragujevac, Kragujevac, Serbia. ${ }^{4}$ Wound Healing Clinical Research Program, \\ University of Miami Hospital, University of Miami Health System, Miami, Florida, USA.
}

\begin{abstract}
Diabetic foot ulcers (DFUs) are a life-threatening disease that often results in lower limb amputations and a shortened life span. Current treatment options are limited and often not efficacious, raising the need for new therapies. To investigate the therapeutic potential of topical statins to restore healing in patients with DFUs, we performed next-generation sequencing on mevastatin-treated primary human keratinocytes. We found that mevastatin activated and modulated the EGF signaling to trigger an antiproliferative and promigratory phenotype, suggesting that statins may shift DFUs from a hyperproliferative phenotype to a promigratory phenotype in order to stimulate healing. Furthermore, mevastatin induced a migratory phenotype in primary human keratinocytes through EGF-mediated activation of Rac1, resulting in actin cytoskeletal reorganization and lamellipodia formation. Interestingly, the EGF receptor is downregulated in tissue biopsies from patients with DFUs. Mevastatin restored EGF signaling in DFUs through disruption of caveolae to promote keratinocyte migration, which was confirmed by caveolin-1 (Cav1) overexpression studies. We conclude that topical statins may have considerable therapeutic potential as a treatment option for patients with DFUs and offer an effective treatment for chronic wounds that can be rapidly translated to clinical use.
\end{abstract}

Conflict of interest: MTC is listed as an inventor of a patent, PCT/ US2010/062361 "Composition and methods for promoting epithelialization and wound closure," issued to New York University based on the data presented, in part, in the study and stands to potentially gain royalties from future commercialization.

Copyright: (c) 2019, American Society for Clinical Investigation.

Submitted: April 3, 2019

Accepted: October 16, 2019

Published: December 5, 2019 .

Reference information: /CI Insight. 2019;4(23):e129320.

https://doi.org/10.1172/jici.

insight.129320.

\section{Introduction}

Diabetic foot ulcers (DFUs) are a debilitating and life-threatening complication of diabetes mellitus (DM) and are a frequent cause of lower limb amputations, exhibiting a 5-year mortality rate greater than 50\% (1-4). Patients with DM often develop several complications that include neuropathy, ischemia, infection, impaired immune function, fibrosis, and vascular complications, all of which have been associated with DFUs and contribute to poor healing outcome $(5,6)$. DFUs are characterized by a hyperproliferative and nonmigratory epidermis, with treatments remaining limited and often not efficacious in restoring healing. Topical statins represent a promising new therapeutic approach for the treatment of chronic wounds, and several studies have shown that statins exert beneficial pleiotropic effects extending beyond reducing systemic cholesterol levels. These include antiinflammatory, antibacterial, anticancer, and improved endothelial function, suggesting that statins may have beneficial effects in the treatment of various diseases (7-14). In addition, statins are currently recommended as a standard of care therapy for DM, and systemic statin use has been shown to correlate with increased wound healing in patients with chronic wounds $(8,15,16)$. We have previously shown that mevastatin promotes wound closure in a human skin ex vivo and porcine in vivo wound models (11). However, the full therapeutic potential and mechanisms by which topical statins promote wound healing remain largely unknown.

In this study, we performed next-generation sequencing on primary human keratinocytes (HEKs) treated with mevastatin to uncover the therapeutic potential and mechanisms of statin action as a potential treatment for chronic wounds. Our results show that statins modulated the EGF pathway, a potent stimulator of keratinocyte migration and wound healing (17), in a way that selectively triggered an antiproliferative and promigratory phenotype in HEKs, suggesting that statins may shift chronic wounds from a hyperproliferative phenotype to a promigratory phenotype. In addition, we found that treatment with 
mevastatin resulted in Rac1 activation and lamellipodia formation to trigger a promigratory phenotype in HEKs. Importantly, we found that EGFR signaling was downregulated in tissue samples obtained from patients with nonhealing DFUs in comparison with patients with acute healthy wounds (AWs) and that treatment with statins resensitized DFUs to EGF signaling through the inhibition of caveolae formation. Overexpression studies of caveolin-1 (Cav1) confirmed that statins restore EGF sensitivity and keratinocyte migration in vitro. Furthermore, we found that treatment with mevastatin inhibited Cav1 levels in porcine in vivo wounds and DFU tissue samples. These data uncover a mechanism of statin action and the therapeutic potential of using already FDA-approved statins as a topical treatment for chronic wounds that are otherwise nonresponsive to standard treatment options by restoring EGFR signaling and facilitating therapeutic reprogramming, which shifts chronic wounds into a healing phenotype.

\section{Results}

In order to understand how statins affect keratinocyte biology that results in accelerated wound healing, we performed RNA-Seq on primary human keratinocytes treated with $5 \mu \mathrm{M}$ mevastatin, the optimal concentration found to stimulate keratinocyte migration (Supplemental Figure 1; supplemental material available online with this article; https://doi.org/10.1172/jci.insight.129320DS1). We examined the differentially expressed genes and identified a mevastatin gene signature with 1988 differentially regulated genes $(P<$ 0.05 ; fold change $>1.5$ ). We found that mevastatin induced 1064 genes and inhibited 924 genes compared with vehicle control (Figure 1A). Gene ontology analysis identified multiple, enriched biological processes that include various aspects of cell migration and proliferation (Figure 1B, top), all processes that are deregulated in DFUs. Ingenuity Pathway Analysis (IPA) revealed several genes from these processes to be involved in the EGF signaling pathway (Figure 1B, bottom), a well-known potent stimulator of proliferation and migration. Specifically, we found that mevastatin inhibited genes involved in cell cycle regulation, such as $C D K 4$ and $C C N B 1$, while simultaneously inducing the expression of genes involved in migration, such as RAC2 and ITGA3 (Figure 1B, bottom, and Supplemental Figure 2). In addition, networks connecting EGFR signaling to downstream biological processes predicted that mevastatin modulated the EGFR signaling pathway to inhibit cell proliferation while stimulating cell migration (Supplemental Figure 2B). This suggests that statins may modulate the EGF pathway to selectively trigger antiproliferative and promigratory phenotypes in keratinocytes.

To further confirm that statins regulate the EGF pathway, we assessed phosphorylated EGFR (pEGFR Y1173), a marker of EGFR activation, and phosphorylated ERK (pERK T202/Y204), a downstream effector of EGF signaling by Western blot in primary human keratinocytes treated with mevastatin. We found that mevastatin induced phosphorylation of both EGFR and ERK (Figure 1C). Next, we confirmed some of the RNA-Seq data by assessing the expression of genes regulated by mevastatin that are involved in EGF signaling by qRT-PCR and Western blot. Mevastatin inhibited several genes involved in proliferation (i.e., $C C N B 1, C C N D 1, C D K 4)$, whereas it induced the expression of several genes involved in migration (i.e., $A R H G E F 1, R A C 2, I T G A 2)$ (Figure 1, D and E), thus further supporting the notion that statins modulate EGF signaling to inhibit proliferation while inducing migration. To assess the effects of statins on EGF signaling pathways and to confirm RNA-Seq data functionally, we treated HEKs with mevastatin, EGF, or a combination of mevastatin/EGF and tested cell migration by in vitro scratch assay and cell proliferation by trypan blue exclusion assay. Mevastatin and EGF promoted migration levels comparably (Figure $1 \mathrm{~F})(n$ $=24, P<0.01, P<0.001$, and $P<0.0001,1$-way ANOVA followed by Holm-Sidak's post hoc test). Interestingly, we found that mevastatin inhibited keratinocyte proliferation (even in the presence of EGF) to levels comparable to PD 0332991 isethionate, a CDK4/6 inhibitor (Figure 1F). These results demonstrate selective activation and modulation of EGF signaling events in a manner that blocks keratinocyte proliferation while promoting migration, suggesting that statins may shift the chronic wound phenotype from hyperproliferative to promigratory in order to promote wound healing.

Stimulation of keratinocytes with EGF results in the rapid activation of Rac1 and the reorganization of actin microfilaments that lead to the formation of lamellipodia and stimulate directional cell migration (18). To test if mevastatin-induced activation of the EGF pathway results in actin cytoskeletal reorganization in HEKs, we performed a Rac1-GTP activation assay in HEKs treated with mevastatin (with vehicle and EGF treatments serving as controls). As expected, we found that EGF treatment led to a robust Rac1-GTP activation (Figure 2A) ( $n=3, P<0.05, P<0.01,1$-way ANOVA followed by Holm-Sidak's post hoc test). Interestingly, we found that mevastatin induced Rac1-GTP activation when compared with 
A

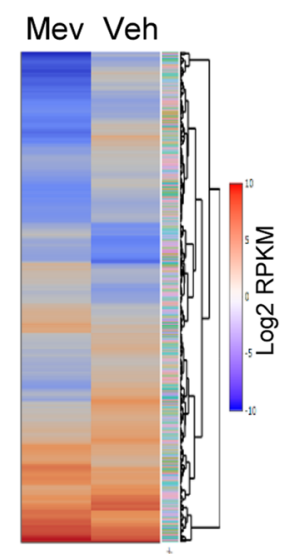

B
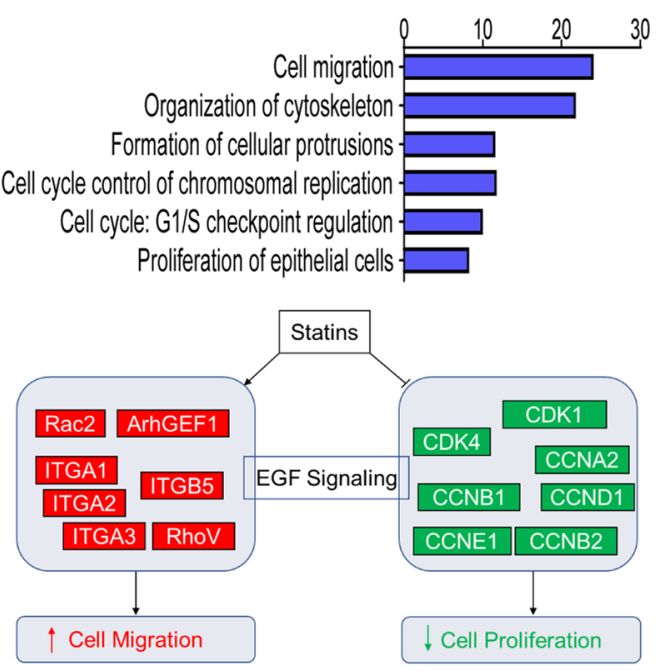

D

Cell migration
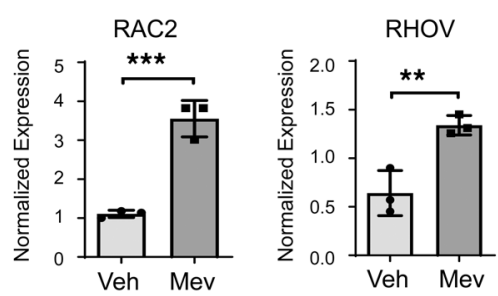

ARHGEF1
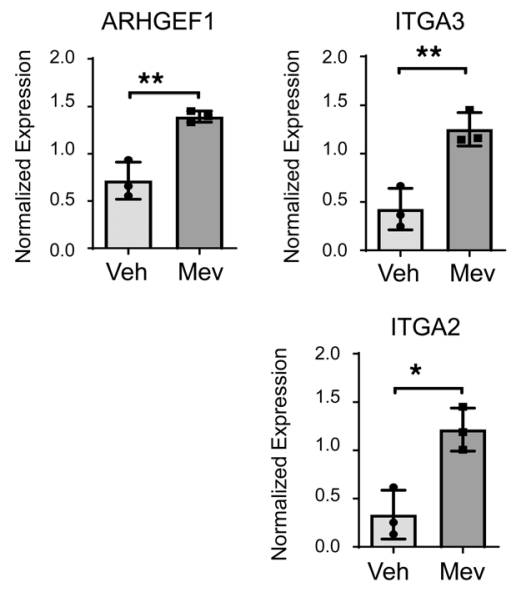

$\mathbf{F}$

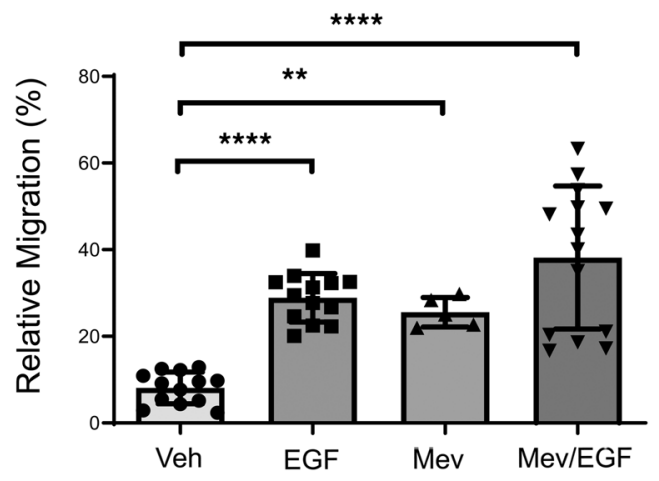

Cell proliferation

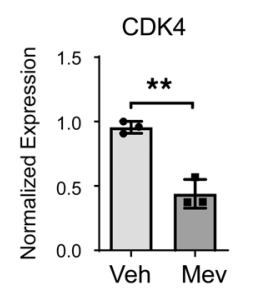

CDK1
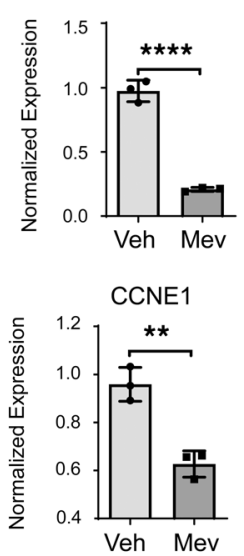

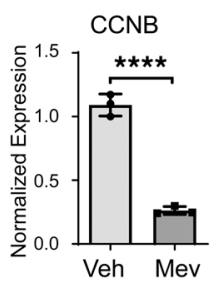

CCNA2
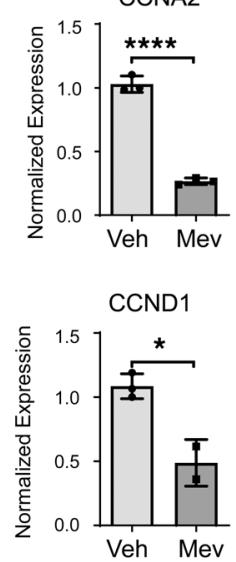
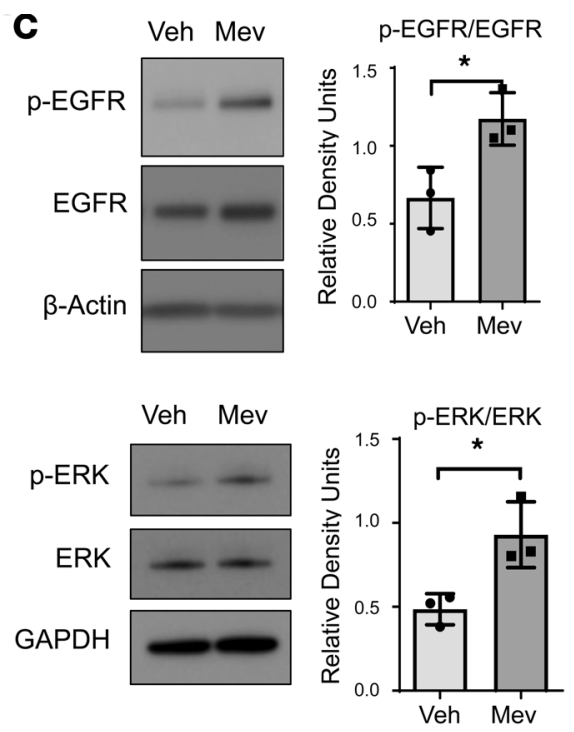

E

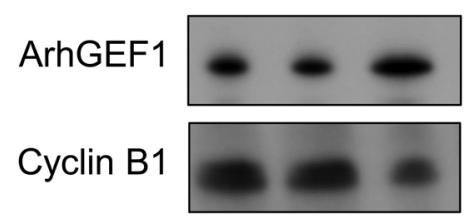

Rac2

$\beta$-Actin

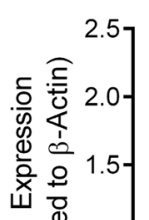

竞 $\frac{N}{\bar{\omega}}$

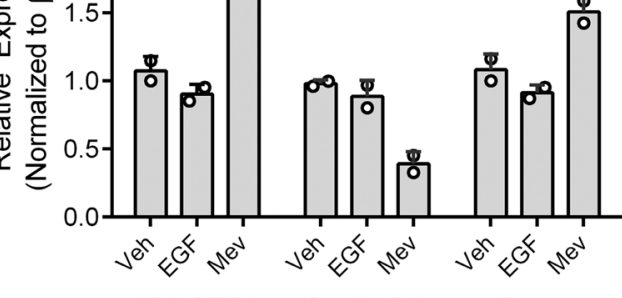

ARHGEF1 Cyclin B1 Rac2

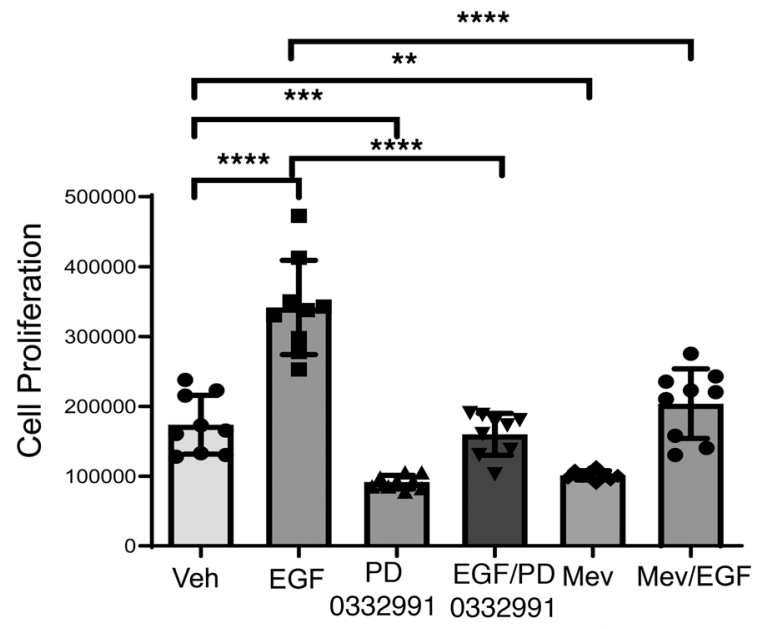


Figure 1. Mevastatin modulates EGF signaling pathway in primary human keratinocytes to inhibit cell proliferation while promoting EGF-induced cell migration. (A) Heatmap of genes regulated by mevastatin in human primary keratinocytes. (B) Gene ontology analysis of biological processes enriched in mevastatin-treated keratinocytes. Diagram of RNA-Seq data showing mevastatin modulation of EGF signaling pathway by inhibiting cell proliferation while inducing cell migration in human keratinocytes (HEKs). Cenes in red indicate mevastatin-induced genes involved in migration and genes in green indicate mevastatin-inhibited genes involved in proliferation. (C) Western blot and quantification of pEGFR (Y1173) and total EGFR and downstream effector pERK and total ERK in HEKs treated with $5 \mu \mathrm{M}$ mevastatin for 48 hours $(n=6)$. Mevastatin significantly induced $p$-EGFR and its downstream effector $p$-ERK. Data are represented as mean \pm SD and were analyzed by Student's $t$ test; ${ }^{*} P<0.05$. (D) Confirmation of RNA-Seq data by $q P C R$ of proliferation and migration genes known to be regulated by EGF signaling in HEKs treated with mevastatin $(n=6)$. Mevastatin inhibited genes involved in cell proliferation and induced genes involved in migration. Data are represented as mean $\pm \mathrm{SD}$ and were analyzed by Student's $t$ test; ${ }^{*} P<0.05,{ }^{* *} P<0.01,{ }^{* * *} P<$ 0.001 , and ${ }^{* * *} P<0.0001$. (E) Western blot and quantification of mevastatin-induced migratory genes (ArhGEF1, Rac2) and proliferation genes (Cyclin B1) suppressed by mevastatin. (F) HEK scratch assay and cell proliferation assay treated in the presence or absence of $25 \mathrm{ng} / \mathrm{mL}$ EGF for 24 hours. $50 \mathrm{nM}$ of PD 0332991, a CDK4 inhibitor, served as a control for cell proliferation assay. Mevastatin stimulated keratinocyte migration while inhibiting cell proliferation even in the presence of EGF. Data are represented as mean \pm SD and were analyzed by a 1-way ANOVA followed by Holm-Sidak's post hoc test, ${ }^{* *} P<0.01$, ${ }^{* * *} P<0.001,{ }^{* * *} P<0.0001$.

vehicle-treated cells, supporting the notion that mevastatin promotes a migratory phenotype through the activation of the EGF-Rac1 signaling pathway.

To test the potential mechanism by which mevastatin induces a migratory phenotype in HEKs, we assessed the actin cytoskeletal reorganization by costaining with phalloidin and ITGB5. We treated HEKs with mevastatin, EGF, or both in combination with tyrphostin AG (a selective EGFR tyrosine kinase inhibitor); vehicle treatment served as a control. As expected, EGF induced prominent actin filaments resembling lamellipodia with robust ITGB5 colocalization, whereas tyrphostin AG 1478 inhibited EGF-induced formation of lamellipodia formation (Figure 2B). Treatment with mevastatin induced lamellipodia formation and ITGB5 colocalization in HEKs, whereas the combination treatment with tyrphostin AG 1478 abolished lamellipodia formation (Figure 2, B and C) $\left(n=3,{ }^{* * * *} P<0.0001,1\right.$-way ANOVA followed by Holm-Sidak's post hoc test), further confirming that mevastatin promotes a migratory phenotype through activation of the EGF-mediated pathway.

Our previous data have shown that EGFR is downregulated and mislocalized in the cytoplasm of venous leg ulcers (19). To test if EGFR signaling is downregulated in DFUs, we obtained tissue samples from patients with DFUs (Supplemental Table 1) and assessed pEGFR (Y1173) by Western blot. AWs served as controls. As expected, we observed a strong induction of pEGFR activation in AWs (Figure 3A). However, we found inhibition of pEGFR in tissue samples from patients with DFUs (Figure 3B), suggesting that the downregulation of the EGFR pathway in DFUs may contribute to the inhibition of wound healing.

To test if mevastatin can restore EGF signaling under the pathological conditions present in DFUs, we treated tissue samples obtained from patients with mevastatin and maintained them at the air-liquid interface for 48 hours. Activation of pEGFR was assessed by Western blotting. We found that the treatment of DFUs with mevastatin strongly induced pEGFR in comparison with control (Figure 3, C and D), demonstrating the efficacy of topical mevastatin treatment to restore EGFR signaling in DFUs and promote healing. Activation of pEGFR in DFUs treated with mevastatin was also confirmed by immunofluorescence staining (Figure 3E).

Findings from our laboratory have shown that Cav1 is induced in DFUs and acts as an inhibitor of wound healing by sequestering and blocking EGFR in a special type of lipid rafts, caveolae (20). To test if mevastatin restores EGF signaling in DFUs by disrupting Cav1 function and thus the formation of caveolae, we assessed Cav1 expression by Western blot in tissue samples obtained from DFUs treated with mevastatin ex vivo. As expected, high levels of Cav1 in vehicle-treated controls were significantly inhibited by mevastatin treatment (Figure 4, A and B). The inhibition of Cav1 by mevastatin was also confirmed in HEKs (Figure 4, C and D). Although RNA-Seq data did not show Cav1 regulation by mevastatin at the transcriptional level, we found that mevastatin inhibited Cav1 at the protein level. This may be due to the dependence of Cav1 on cholesterol since depletion of cholesterol has been shown to result in Cav1 protein degradation and disruption of caveolae (21).

We further assessed Cav1 levels in mevastatin-treated DFUs by immunostaining. Histological analysis of DFUs demonstrated hyperproliferative epidermis with a thickened cornified layer, which is a hallmark of DFUs (ref. 1 and Supplemental Figure 3A). We found an inhibition of Cav1 in DFUs treated with mevastatin (Supplemental Figure 3B). Furthermore, we assessed Cav1 expression by immunoperoxidase staining in porcine wounds treated with mevastatin. Interestingly, we found that mevastatin treatment inhibited Cav1 levels and promoted epithelialization compared with vehicle control (Supplemental Figure 3C). 


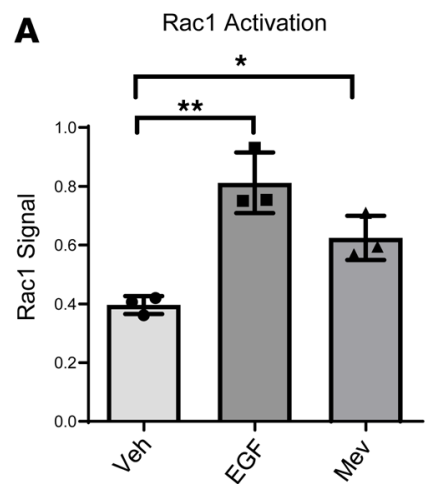

B

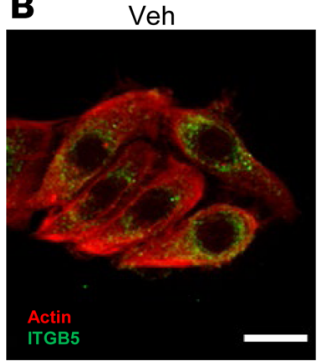

AG1478
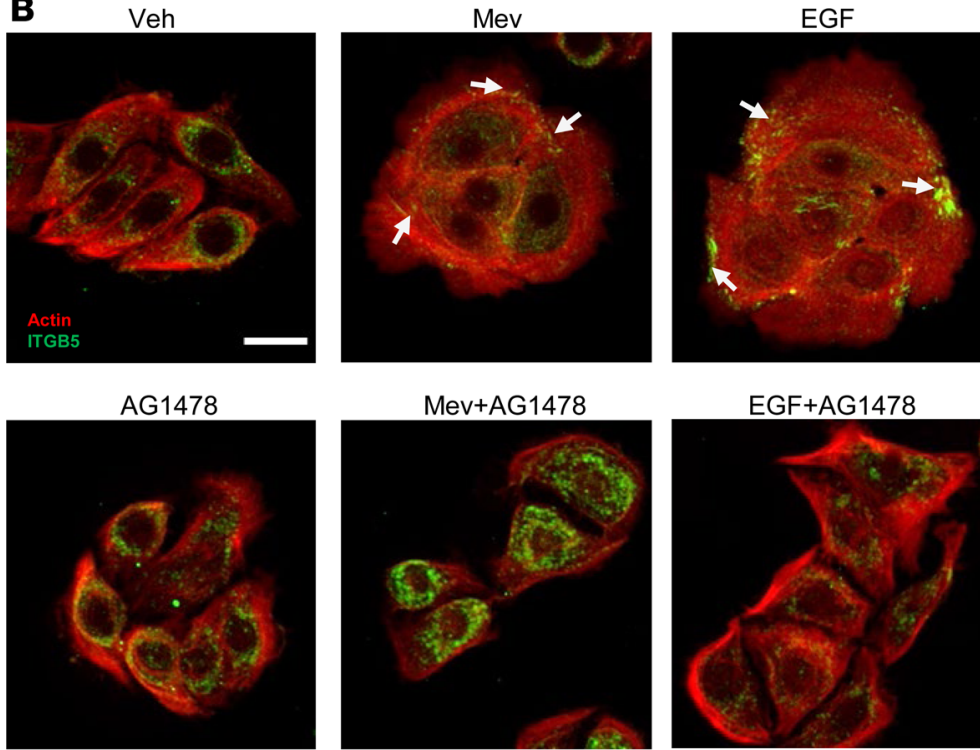

Figure 2. Mevastatin activates the EGF pathway to induce actin cytoskeletal reorganization to promote a migratory phenotype. (A) G-LISA Rac1-GTP activation assay in HEKs treated with 5 $\mu \mathrm{M}$ mevastatin for 48 hours $(n=6)$. Treatment with 12.5 $\mathrm{ng} / \mathrm{mL}$ EGF for 5 minutes served as positive control. Mevastatin induced Rac1-GTP activation. (B) ITCB5 and phalloidin staining of human keratinocytes (HEKs) treated with mevastatin or EGF in the presence or absence of 150 nM tyrphostin AG 1478. Scale bar: $10 \mu \mathrm{m}$. LP, lamellipodia. (C) Percentage of ITCB5+ lamellipodia ${ }^{+}$cells $(n=3)$. Mevastatin induced lamellipodia formation, whereas tyrphostin AG 1478 inhibited mevastatin-induced lamellipodia. Data are represented as mean $\pm \mathrm{SD}$ and were analyzed by a 1-way ANOVA followed by Holm-Sidak's post hoc test; ${ }^{* * * *} P<0.0001$.

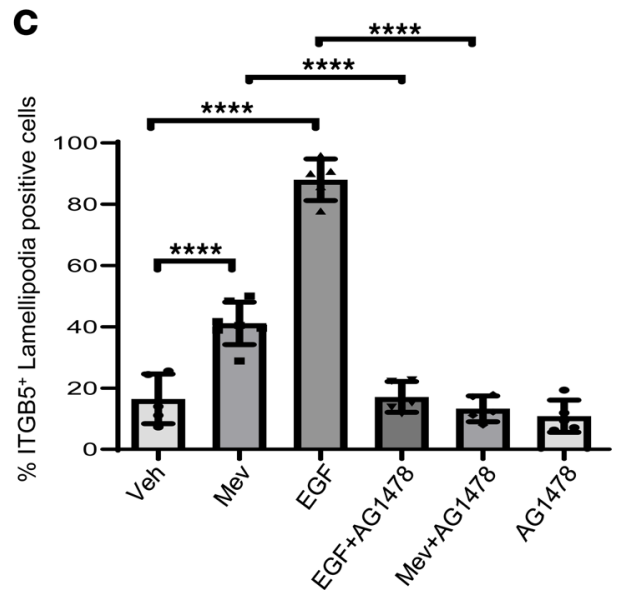

These results demonstrate that inhibition of Cav1 protein levels by mevastatin may restore EGFR signaling to promote keratinocyte migration

Since EGFR interaction with Cav1 and localization to caveolae has previously been shown to antagonize EGFR signaling (20), we performed sucrose gradient fractionation to determine if mevastatin inhibits the localization of Cav1 to the plasma membrane and thus inhibits the formation of caveolae. To test this, we treated HEKs with mevastatin for 48 hours and performed subcellular fractionation. Vehicle treatment served as a control and E-cadherin as a marker for the plasma membrane. As expected, Cav1 was found in membrane, E-cadherin-positive fractions (Figure 4E). Mevastatin treatment inhibited the localization of Cav1 to membranous fractions, suggesting that mevastatin-mediated restoration of 
A
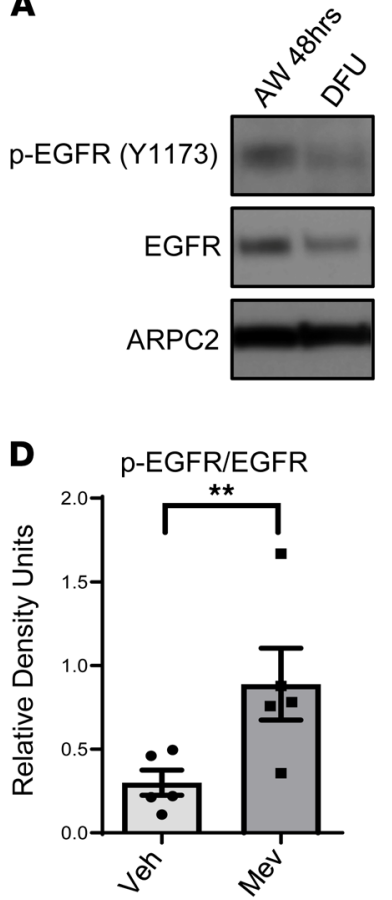

B

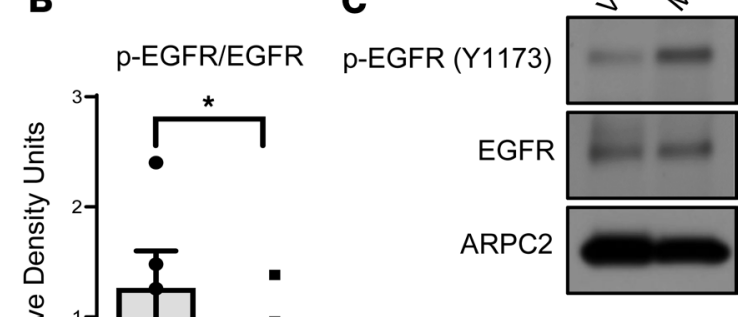

Figure 3. Mevastatin restores EGF activation in diabetic foot ulcers. (A and B) Western blot and quantification of pEGFR (Y1173) and total EGFR in acute healthy wounds (AWs) $(n=5)$ and diabetic foot ulcers (DFUs) $(n=6)$. pEGFR is downregulated in DFUs compared with AWs. Data are represented as mean \pm SEM and were analyzed by Student's $t$ test; ${ }^{*} P<0.05$. (C and $\mathbf{D}$ ) Western blot and quantification of $p$-EGFR and total EGFR from samples obtained from the nonhealing edge of patients with DFUs treated with 5 $\mu \mathrm{M}$ mevastatin for 48 hours $(n=5)$. Mevastatin significantly induced pEGFR in samples obtained from the nonhealing edge of DFUs compared with vehicle-treated control. Data are represented as mean \pm SEM and were analyzed by a ratio-paired $t$ test; ${ }^{*} P<0.01$. (E) Immunofluorescence staining of $p E G F R$ in mevastatin-treated DFUs. Mevastatin strongly induced $p$-EGFR compared with vehicle-treated control. Scale bar: $100 \mu \mathrm{m}$.

E

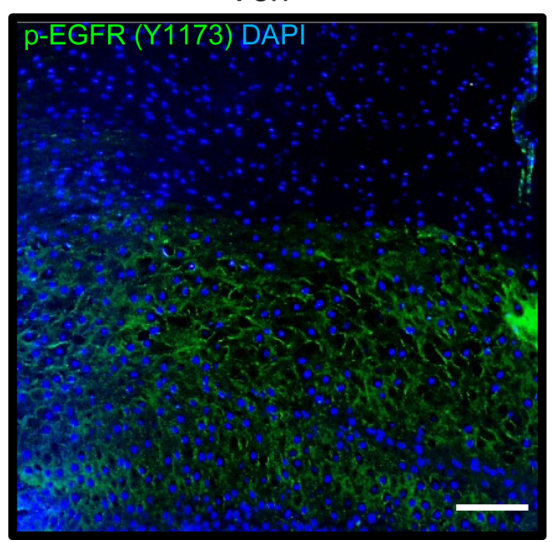

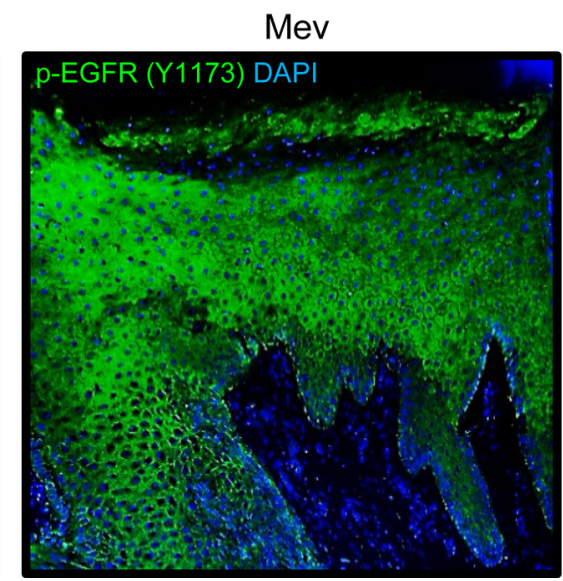

EGFR activation may be occurring through disruption of caveolae formation.

To test whether statins can reverse caveolae inhibition of keratinocyte migration, we overexpressed Cav1 $\left(\mathrm{Cav1}^{\mathrm{O} / \mathrm{E}}\right)$ to induce caveolae formation in $\mathrm{HaCaT}$ keratinocyte cell line and performed an in vitro keratinocyte wound scratch assay. HEKs were treated with mevastatin, EGF, or a combination of both; empty vector (EV) served as control. Overexpression of Cav1 was confirmed by Western blot (Figure 4F). In addition, we found that treatment with mevastatin downregulated levels of Cav1 in Cav1 ${ }^{\mathrm{O} / \mathrm{E}}$ cells (Figure 4F). Moreover, Cav1 ${ }^{\mathrm{O} / \mathrm{E}}$ cells showed downregulated levels of migratory markers (ArhGEF1, Rac2) compared with EV cells, whereas treatment with mevastatin reversed Cav1-mediated inhibition of migratory markers (Supplemental Figure 3). Quantification of keratinocyte scratch assay showed that the overexpression of Cav1 inhibited keratinocyte migration even in the presence of EGF (Figure 4G), demonstrating decreased sensitivity to EGF, similar to that observed in chronic wounds. Interestingly, treatment with mevastatin reversed Cav1-mediated inhibition of keratinocyte migration and was able to restore EGF sensitivity when used in combination with EGF (Figure 4G) $(n=24, P<0.05, P<0.0001$, 1-way ANOVA followed by Holm-Sidak's post hoc test), demonstrating that statins disrupt caveolae formation to restore EGF signaling and promote keratinocyte migration. 
A

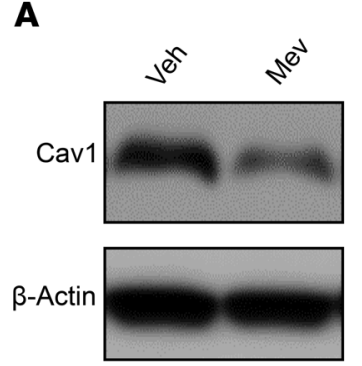

E

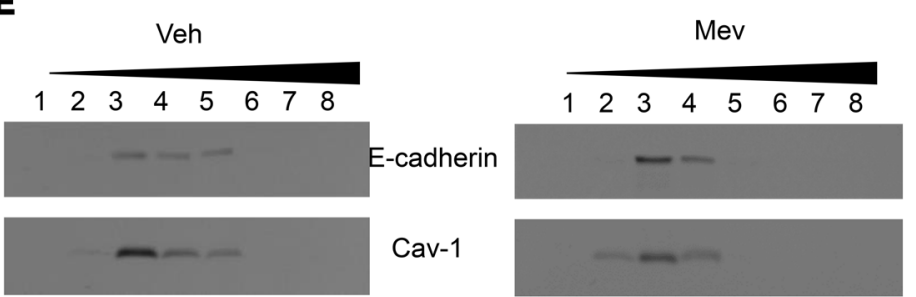

F

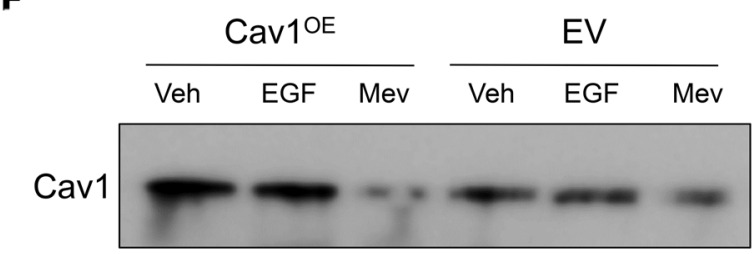

$\beta$-Actin
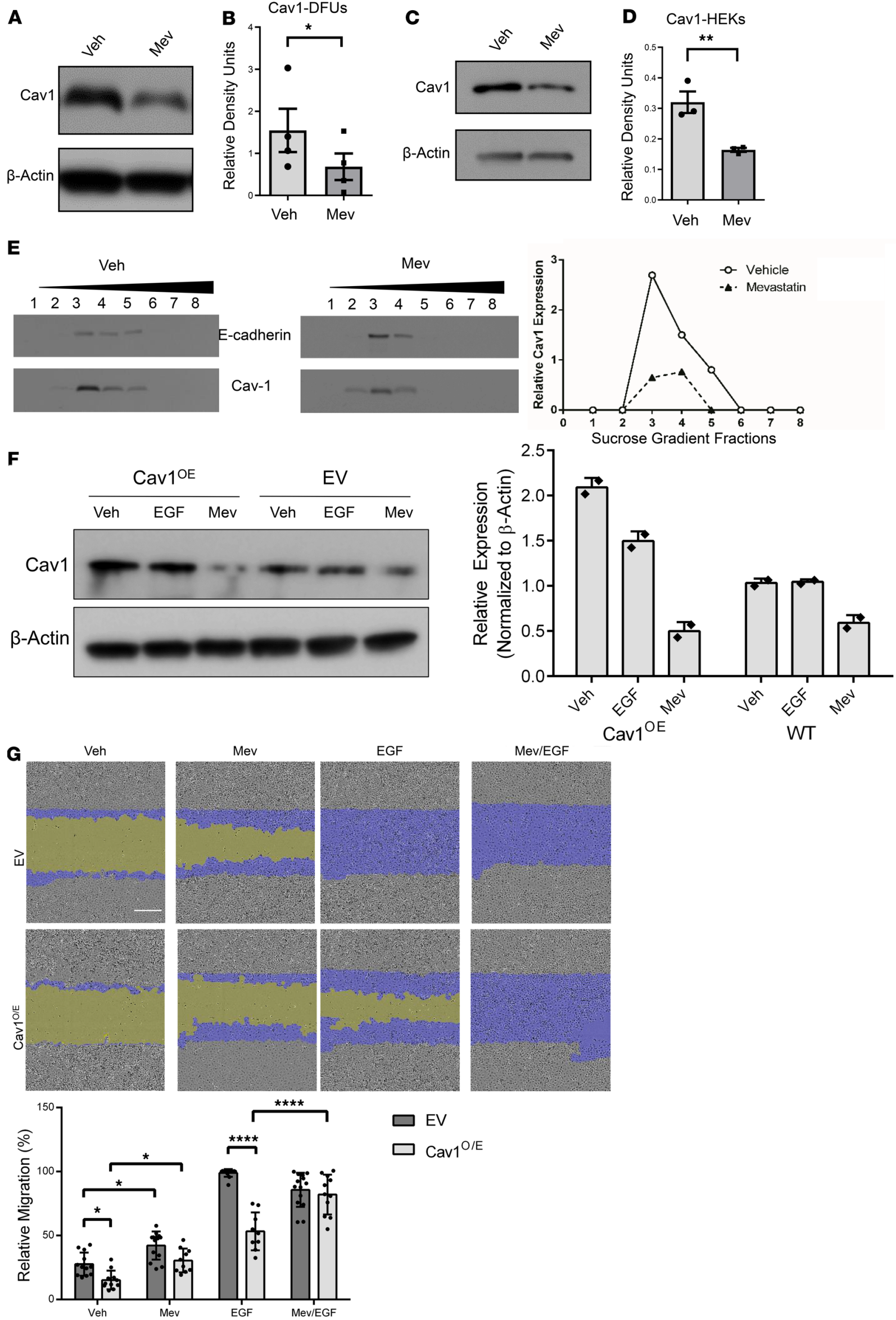

D Cav1-HEKs

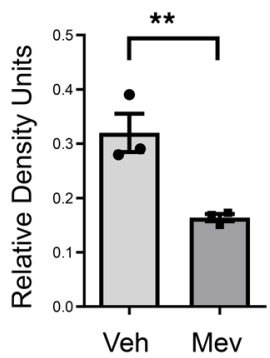


Figure 4. Mevastatin disrupts caveolae through inhibition of caveolin-1 to restore EGF signaling and keratinocyte migration. (A and B) Western blot and quantification of caveolin-1 (Cav1) in samples obtained from nonhealing edge of diabetic foot ulcers (DFUs) treated with $5 \mu M$ mevastatin for 48 hours ( $n=$ 4). Mevastatin significantly inhibited Cav1 compared with vehicle-treated control. Data are represented as mean \pm SEM and a paired $t$ test was performed; ${ }^{*} P<0.05$. (C and D) Western blot and quantification of Cav1 in human keratinocytes (HEKs) treated with $5 \mu$ M mevastatin for 48 hours $(n=3$ ). Mevastatin significantly inhibited Cav1 compared with vehicle control. Data are represented as mean \pm SD and were analyzed by Student's $t$ test; ${ }^{* *} P<0.01$. (E) Sucrose gradient of Cav1 from mevastatin-treated HEKs $(n=2)$. E-cadherin served as a marker for the plasma membrane. Quantification of Cav1 to E-cadherin in gradient fractions demonstrates mevastatin inhibited Cav1 in plasma membrane fractions compared with vehicle-treated control. (F) Western blot and quantification of Cav1 in Cav1 ${ }^{0 / E}$ cells. Treatment with mevastatin inhibited Cav1. (C) Scratch assay of HaCaTs overexpressing Cav1 treated with $5 \mu \mathrm{M}$ mevastatin in the presence or absence of $25 \mathrm{ng} / \mathrm{mL}$ EGF for 24 hours $(n=6)$. Cav1 overexpression inhibited keratinocyte migration even in presence of EGF, whereas treatment with mevastatin restored keratinocyte migration and EGF-induced migration. Data are represented as mean \pm SD and were analyzed by a 1-way ANOVA followed by Holm-Sidak's post hoc test; ${ }^{*} P<0.05,{ }^{* * *} P<0.0001$.

\section{Discussion}

Chronic DFUs are a serious life-threatening disease; they are rapidly increasing in incidence and often result in lower limb amputation and a shortened life span $(2,22-24)$. To date, treatments for DFUs remain limited and achieve modest efficacy in restoring healing (1). A major area of attention has been given to using biologically active recombinant growth factors (such as EGF) to stimulate wound healing as treatments for chronic wounds; however, several attempts have demonstrated limited success. We performed next-generation sequencing on human keratinocytes treated with statins to uncover the full therapeutic potential and mechanism(s) of statin action as a treatment for chronic wounds. Our results demonstrate that statins modulate the EGF pathway in a way that inhibits proliferation and promotes migration in HEKs, suggesting that statins may shift chronic wounds from a hyperproliferative phenotype to a promigratory phenotype. Importantly, we found EGFR signaling to be downregulated in tissue samples obtained from patients with DFUs compared with patients with AWs and that treatment with statins resensitized DFUs to EGF signaling by inhibiting Cav1 localization to the cell membrane and the subsequent formation of caveolae, suggesting that disruption of caveolae formation may restore EGF sensitivity in patients with chronic wounds. These results demonstrate the therapeutic potential of using already FDA-approved statins as a treatment for chronic wounds that are otherwise nonresponsive to standard treatment.

Epithelialization is a defining parameter of wound healing and relies on proper execution of keratinocyte migration to close the wound (25). The epidermis of DFUs has reduced expression of migratory markers and increased proliferation markers that contribute to reduced capacity for keratinocyte migration (25). This inhibition of migration may originate from the interaction of Rac1 with Cav1. Rac1 is known to facilitate the formation of lamellipodia, a process that is integral to directional cell movement and has been shown to interact with Cav1 $(26,27)$. Our observation that mevastatin stimulates cell migration may be a result of the increased activation of Rac1 via the upregulation of ArhGEF1, a known guanine nucleotide exchange factor for Rac1 (Figure 1 and Supplemental Figure 4). We have also previously shown that induced and deregulated cortisol levels in chronic wounds induced c-Myc expression and contribute to a hyperproliferative, nonmigratory phenotype (20). In addition, we have previously shown that Cav1 is induced in DFUs (20) and antagonizes signaling molecules necessary for proper keratinocyte migration. All these factors result in a nonmigratory epidermis that fails to restore the barrier and contributes to the pathophysiology of DFUs.

One potential mechanism for the upregulation of Cav1 in DFUs is through the upregulation of cortisol. We have previously shown that cortisol is upregulated at the wound edge of nonhealing chronic wounds (20). Since skin is a site of extrahepatic cholesterol synthesis and extraadrenal cortisol synthesis, modulation of cholesterol and cortisol production can directly affect Cav1 and its control of wound closure (28-31). For example, it has been shown that free cholesterol upregulates Cav1 mRNA (via 2 SREBP-binding elements located in the Cav1 promoter region) and stabilizes Cav1 protein $(32,33)$. Thus, it should be of no surprise that disruption of cholesterol synthesis by statins, or removal of cholesterol by cyclodextrins, leads to disruption of caveolae, as well as destabilization, ubiquitination, and subsequent degradation of Cav1 protein in the lysosomes (21). Our findings are in line with those of previous groups, which have shown that Cav1 can sequester and antagonize functioning of numerous growth factor receptors, as well as various intracellular signaling molecules in what has been proposed as the caveolin signaling hypothesis (34-37). Thus, is it not surprising that disruption of caveolae would result in activation of the EGFR signaling, as evidenced by our current study as well as others, albeit in different cell types (38, 39). Although we did not find Cav1 to be regulated at the transcriptional level by statins, the inhibition of Cav1 at the protein level by statins is most likely due to cholesterol depletion and disruption of caveolae at the plasma mem- 
brane, as was confirmed by sucrose gradient fractionation. Since this mechanism is conserved in various tissues $(38,39)$, this could explain some of the pleiotropic effects observed with statin treatment and raise possibilities for the use of statins for the treatment of a plethora of disorders.

Growth factors play a significant role in coordinating the wound healing process, thus suggesting growth factor therapy as a possible course of treatment. However, clinical trials testing growth factors as potential therapies showed limited success (40). One proposed reason for the repetitive failures is the high degree of protease activity in chronic wounds, resulting in the rapid degradation of an exogenously applied growth factor (1). In addition, the deregulation of several key signaling pathways that include the EGF and TGF- $\beta$ pathways results in the inability of keratinocytes to respond to these signaling molecules even when applied exogenously $(5,9,19,41,42)$. This was ultimately due to a lack of knowledge of the molecular mechanisms of chronic wounds at the time. Later studies by Brem et al. found that the EGFR was downregulated and mislocalized in the cytoplasm in venous leg ulcers, thus rendering keratinocytes at the nonhealing edge unresponsive to EGF stimulation $(5,19)$. Findings presented in this manuscript provide mechanistic insight and an explanation for why clinical trials with recombinant EGF in patients with chronic wounds showed limited success. Therapies aimed at inhibiting proliferation and inducing migration, such as topical statins, offer a unique advantage for the treatment of chronic wounds that are characterized by a hyperproliferative, nonmigratory epidermis. Furthermore, the restoration of EGF signaling by statins provides a basis for multiple treatment approaches of topical statin-growth factor combinatorial therapy in order to achieve maximum stimulation of healing in patients with chronic wounds. Our data suggest that statins may facilitate therapeutic reprogramming of the nonhealing into an acutely healing phenotype to restore healing in patients with DFUs.

\section{Methods}

RNA-Seq and IPA analysis. Preparation and sequencing of RNA libraries was carried out in the John P. Hussman Institute for Human Genomics Center for Genome Technology. Briefly, total RNA was quantified and qualified using the Agilent Bioanalyzer and found to have an RNA integrity score of 10 . We used 500 ng total RNA as input for the Illumina TruSeq Stranded Total RNA Library Prep Kit with Ribo-Zero to create ribosomal RNA-depleted sequencing libraries. Each sample had a unique barcode to allow for multiplexing and was sequencing to greater than 80 million raw reads in a single-end 75-bp sequencing run on the Illumina NextSeq500.

Raw sequence data were processed by the on-instrument Real Time Analysis software (v.2.4.11) to base call files. These were converted to demultiplexed FASTQ files with the Illumina-supplied scripts in the BCL2FASTQ software (v2.17). We used FASTQC software (http://www.bioinformatics.babraham.ac.uk/projects/fastqc/) to determine the quality of the reads (i.e., per base sequence quality, duplication rates, and overrepresented k-mers). Illumina adapters were trimmed from the ends of the reads using Trim Galore! package (http://www.bioinformatics.babraham.ac.uk/projects/trim_galore/). Reads were aligned to the human reference genome (hg19) with the STAR aligner (v2.5.0a). Gene count quantification for total RNA was performed using the GeneCounts function within STAR against the GENCODE v19 human transcript.gtf file.

Gene count data were input into edgeR software for differential expression analysis. Briefly, gene counts were normalized using the trimmed mean of $\mathrm{M}$-values method to account for compositional difference between the libraries. Group differential expression was calculated using the exact test implement in edgeR. The expression profiles were deposited in the Gene Expression Omnibus database (GSE137441).

Pathway analysis was conducted using IPA software (QIAGEN Inc.; www.qiagenbioinformatics.com/ products/ingenuity-pathway-analysis/), which used Fisher's exact test to detect significantly enriched pathways and biological processes, with a $P$ value of 0.05 or less considered significant.

Cell culture and wound scratch assay. Primary cultures of adult normal HEKs were isolated as described previously (43). HEKs were maintained in a serum-free keratinocyte medium supplemented with EGF and bovine pituitary extract (Keratinocyte-SFM, LifeSci, 10724-011). HEKs were grown to confluence in 96-well ImageLock plates (Essen Bioscience), treated with $4 \mu \mathrm{g} / \mathrm{ml}$ Mitomycin-C (Sigma-Aldrich), and wounded by scratch with a 96-pin wound-making tool (WoundMaker, Essen Bioscience). Cells were incubated for 24 hours and 2 representative images from each well of relative migration were taken every 2 hours after the initial wound using IncuCyte Zoom system (Essen Bioscience) and quantified using Cell Migration Analysis software module (Essen Bioscience). Treatments were carried out as follows: $25 \mathrm{ng} / \mathrm{ml}$ EGF, $5 \mu \mathrm{M}$ mevastatin (Sigma-Aldrich, M2537), or both in combination. For HaCaT cells (a gift from Joa- 
quin Jimenez, University of Miami Miller School of Medicine, Miami, Florida, USA), Cav1 overexpressing $\mathrm{HaCaT}$ cells were switched to DMEM media containing $0.1 \%$ FBS and $1 \times$ Penicillin/Streptomycin/ Glutamine for 24 hours prior to scratch assay.

Skin specimens. Discarded, unidentified, DFU samples ( $n=6$, Supplemental Table 1$)$ were obtained from consenting patients receiving standard care and considered as nonhuman subject research by the University of Miami Institutional Review Board. Patient demographics and sample characteristics are included in Supplemental Table 1. Ulcers did not have any clinical signs of infection. Specimens from each patient were maintained at the air-liquid interface and treated with either vehicle (ethanol) or $5 \mu \mathrm{M}$ mevastatin 3 times daily for 48 hours. Samples were harvested for protein assessments (Western blot) and immunostaining.

Human skin ex vivo wound model. Discarded human skin tissue samples were obtained from patients with DFUs undergoing voluntary reduction surgeries at the University of Miami Hospital and were found to be exempt from human subject research under 45 CFR 46.101 .2 by the Institutional Review Board at the University of Miami Miller School of Medicine. Healthy human skin samples were used to generate acute wounds as previously described $(28,41,44)$. Briefly, a 3-mm punch (Acuderm) was used to make wounds in the epidermis through the reticular dermis, and 3-mm discs of epidermis were excised using sterile scissors. Skin discs $(6 \mathrm{~mm})$ with the 3-mm epidermal wound in the center were immediately transferred to the air-liquid interface with DMEM (BioWhittaker) and supplemented with antibiotic/antimycotic and fetal bovine serum (Gemini Bio-Products). Wounded skin specimens were maintained the air-liquid interface at $37^{\circ} \mathrm{C}$ in a humidified atmosphere of $5 \% \mathrm{CO}_{2}$.

Porcine in vivo wound model. Five young, female, specific pathogen-free pigs (Ken-O-Kaw Farms) weighing between $25 \mathrm{~kg}$ and $35 \mathrm{~kg}$ were used as previously described (11). The experimental animal protocols were approved by the University of Miami Institutional Animal Care and Use Committee (protocol 13-140). The methods for animal preparation and wounding were explained in detail in our previous study (11). Briefly, partial-thickness wounds ( $\mathrm{mm} \times 10 \mathrm{~mm} \times 0.5 \mathrm{~mm}$ deep) were made on the paravertebral area using a modified electrokeratome. The wounds were separated from one another by approximately $50-\mathrm{mm}$ areas of unwounded skin. Wounds were treated within 20 minutes (or when hemostasis has been achieved) after creation with either vehicle (ethanol in PBS) or $250 \mu \mathrm{M}$ mevastatin. At least 6 wounds from each group per animal were used for histological evaluation.

Cell proliferation assay. HEKs were seeded in 6-well plates at 50,000 cells/well and switched to basal media for 24 hours and treated with $5 \mu \mathrm{M}$ mevastatin, $25 \mathrm{ng} / \mathrm{ml}$ EGF, $50 \mathrm{nM}$ PD 0332991 isethionate (Sigma-Aldrich), or both in combination for 48 hours, with each condition carried out in triplicates. Cells were trypsinized and mixed with trypan blue and live cells were counted with a hemocytometer. Experiments were carried out in triplicates.

Phalloidin staining. HEKs were grown to approximately 30\% confluency and switched to basal media for 24 hours and treated with $5 \mu \mathrm{M}$ mevastatin, $150 \mathrm{nM}$ tyrphostin AG 1478 (Cell Signaling Technology), or both in combination, and $12.5 \mathrm{ng} / \mathrm{ml}$ EGF treatment for 5 minutes served as a positive control. Cells were washed with $1 \times$ PBS and then fixed with $4 \%$ paraformaldehyde for 10 minutes at room temperature followed by washing 3 times with PBS. Cells were then permeabilized with $0.1 \%$ Triton X-100 for 5 minutes and washed 3 times with PBS. Cells were stained with ITGB5 (D24A5) (rabbit, 1:100; Cell Signaling Technology; 3629S) overnight at $4^{\circ} \mathrm{C}$ and washed 3 times with PBS. Cells were visualized with Alexa Fluor 488-conjugated goat anti-rabbit antibody (1:300; Invitrogen; A11008) and incubated with $100 \mathrm{nM}$ Actistain 555 phalloidin (Cytoskeletal) for 1 hour followed by washing 3 times with PBS. Cells were analyzed using a Zeiss LSM780 confocal microscope and images were acquired with Zen software. Quantification was carried out by determining the ratio of the number of $\mathrm{ITGB}^{+}$lamellipodia ${ }^{+}$cells to the total number of cells to determine percentage of ITGB5 ${ }^{+}$lamellipodia ${ }^{+}$cells.

Rac1-GTP activation assay. HEKs were treated as described above. Rac1-GTP levels were analyzed using the G-LISA Rac1 Activation Assay Biochem Kit (Cytoskeleton) according to the manufacturer's instructions and with a Bio-Rad Benchmark Plus Microplate spectrophotometer (Bio-Rad). Rac1-GTP levels were normalized to total protein concentration.

Sucrose gradient. HEKs were seeded in $10-\mathrm{cm}^{2}$ dishes and grown to approximately $85 \%$ confluency. Cells were switched to basal keratinocyte serum-free medium for 24 hours and treated with $5 \mu \mathrm{M}$ mevastatin and vehicle served as a control. Cells were washed 3 times with ice-cold PBS and lysed in $25 \mathrm{mM}$ Tris- $\mathrm{HCl} \mathrm{pH}$ 7.5, $150 \mathrm{mM} \mathrm{NaCl}, 5 \mathrm{mM}$ EDTA, 1\% Triton X-100, and Protease/Phosphatase Inhibitor Cocktail (Cell Signaling Technology). A discontinuous density gradient of $0 \%$ to $35 \%$ was prepared using OptiPrep (Sig- 
ma-Aldrich) with $1 \mathrm{mg}$ lysate. The lysates were subjected to ultracentrifugation for 18 hours at $176,849 \mathrm{~g}$ using a Beckman 42Ti rotor and fractions were collected and assessed by Western blot as described below.

Immunostaining. Formalin-fixed, paraffin-embedded tissue sections (each 5- to 7- $\mu$ m thick) were deparaffinized, rehydrated, and H\&E stained or processed for immunostaining as previously described (20). For immunofluorescence staining, tissue sections of discarded DFUs were treated with mevastatin as described above and used for staining with anti-phospho-EGFR (Y1173) (53A5) (rabbit, 1:100; Cell Signaling Technology; 4407) or anti-Cav1 (D46G3) (rabbit, 1:200; Cell Signaling Technology; 3267). pEGFR (Y1173) and Cav1 staining was visualized with Alexa Fluor 488-conjugated goat anti-rabbit antibody (1:500; Invitrogen; A11008) and mounted with Prolong DAPI Gold antifade reagent (Invitrogen) to visualize cell nuclei. Tissue sections of porcine wounds were stained with anti-Cav1 antibody (rabbit, 1:200; Cell Signaling Technology; 3267) diluted in $1 \%$ normal goat serum with $0.3 \%$ Triton X-100, followed by Rabbit-on-Farma HRP-Polymer (Biocare Medical) and then developed using Betazoid DAB Chromogen Kit (Biocare Medical). Images were acquired by using Keyence BZ-X700 Fluorescence microscope and analyzed by NIH Image J software.

Western blot. Extracts for immunoblotting were prepared from a subconfluent normal HEK or wound tissue. Cell extracts were separated on $4 \%-20 \%$ Criterion TGX precast gels (Bio-Rad) and transferred onto PVDF membranes (Bio-Rad). Membranes were blocked with 5\% bovine serum albumin in PBS containing $0.1 \%$ Tween-20, and then incubated with anti-phospho-EGFR (Y1173) (rabbit, 1:1,000; Santa Cruz; sc-12351), anti-EGFR (rabbit, 1:1000; Santa Cruz; sc-03), anti-phospho-ERK1 (T202/Y204) (rabbit, 1:1000; Santa Cruz; sc-7338), ERK (rabbit, 1:5000; Santa Cruz; sc-94), anti-Cav1 (rabbit, 1:1,000; Santa Cruz; sc-894), anti-E-Cadherin (clone 36) (mouse, 1:10,000; BD Transduction Laboratories; 610182), anti-ArhGEF1 (D25D2) (rabbit, 1:1000; Cell Signaling Technology; 3669), anti-Rac2 (goat, 1:1000; Abcam; ab2244), and anti-Cyclin B1 (V152) (mouse, 1:1000; Abcam; ab72). For loading control, we used anti- $\beta$-actin antibody (mouse, 1:10,000; Sigma-Aldrich; A5441), anti-GAPDH (rabbit, 1:1000; Santa Cruz; sc-25788), or anti-ARPC2 (rabbit, 1:5,000; Abcam; ab133315). Representative Western blots of 3 independent experiments were scanned and quantified using NIH ImageJ software.

$R T-P C R$. cDNA was synthesized using 100 ng total RNA using the qScript cDNA Synthesis kit (Quantabio, 95047-100). Real-time PCR was performed in triplicates with 1 ng cDNA per reaction using the CFX96 qPCR thermal cycler and detection system (Bio-Rad) and PerfeCTa SYBR Green Supermix (Quantabio). Relative expression was normalized for levels of actin related protein $2 / 3$ complex subunit 2 (ARPC2). The primer sequences used were: $A R P C 2$, forward (5'-TCCGGGACTACCTGCACTAC-3') and reverse (5'-GGTTCAGCACCTTGAGGAAG-3'); CDK4, forward (5'-TTGGCAGCTGGTCACATGGT-3') and reverse (5'-TCAGATCAAGGGAGACCCTCAC-3'); CCNB1, forward (5'-TGGTGAATGGACACCAACTCT-3') and reverse (5'-TAGCATGCTTCGATGTGGCA-3'); CDK1， forward (5'-CTTGGCTTCAAAGCTGGCTC-3') and reverse (5'-GGGTATGGTAGATCCCGGCT-3'); CCNA2, forward (5'-CCTGCAAACTGCAAAGTTGA-3') and reverse (5'-AAAGGCAGCTCCAGCAATAA-3'); CCNE1, forward (5'-CCCATCATGCCGAGGGAG-3') and reverse (5'-TATTGTCCCAAGGCTGGCTC-3'); CCND1, forward (5'-ATGCCAACCTCCTCAACGAC-3') and reverse (5'-TCTGGTTCCTCGCAGACCTCC-3'); RAC2, forward (5'-CAACGCCTTTCCCGGAGAG-3') and reverse (5'-TCCGTCTGTGGATAGGAGAGC-3'); RHOV, forward (5'-CCTCATCGTCAGCTACACCTG-3') and reverse (5'-GAACGAAGTCGGTCAAAATCCT-3'); ARHGEF1, forward (5'-GATGCAACATACCATCTCTACCG-3') and reverse (5'-CCCCGACTTCTTGTCTCCAC-3'); ITGA3, forward (5'-ATATGGGGCGCGGTGTTCTTG-3') and reverse (5'-CTGCCACCCATCATTGTTCAG-3'); ITGA2, forward (5'-TCATTCTCCCTGCCGGTTG-3') and reverse (5'-TGGAAACTGAGAGACGCCTG-3')

Plasmids and transfections. Cav1 cDNA was directionally cloned into pEGFP-N3 plasmid using NheI and BamHI restriction enzymes and the Quick Ligation Kit (New England BioLabs). Plasmids were confirmed by sequencing. HaCaT cells were plated on 6-well plates at 300,000 cells/well. HaCaT cells were transfected the next day using Attractene transfection reagent (QIAGEN Inc.). A combination of $2 \mu 1$ Attractene and $5 \mu \mathrm{g}$ plasmid DNA was added per well in Opti-MEM I Reduced Serum Media (Gibco), and the complex was allowed to form at room temperature for 15 minutes. HaCaT cells were switched to fresh media with DMEM, 10\% FBS, and $1 \times$ Penicillin/Streptomycin/Glutamine and $60 \mu 1$ of the complex was added per well. Next day, HaCaT cells were switched to fresh growth media for 48 hours followed by selection with $1 \mathrm{mg} / \mathrm{mL} \mathrm{G} 418$ for 2 weeks. Cells were then plated for scratch assay as previously described (20).

Statistics. Statistical analyses were performed using a 1-way ANOVA followed by Holm-Sidak's post hoc test. A 2-tailed Student's $t$ test or a ratio-paired $t$ test was used where indicated. A $P$ value of 0.05 or less was considered significant. 
Study approval. Skin specimens from patients suffering from DFUs were obtained as discarded tissue (specimens that would have otherwise been discarded were obtained from debridement procedures) at the University of Miami Hospital and, as such, were found to be exempt under 45 CFR 46.101.2 by the Institutional Review Board at the University of Miami Miller School of Medicine. Specimens did not contain any of the 18 identifiers noted in the privacy rule and therefore no informed consent was obtained.

\section{Author contributions}

APS, IJ, MTC, RCS, and IP designed the research. AS, IJ, RCS, IP, and GDG, ANE performed the research. RSK contributed the reagents. APS, IJ, RCS, IP, OS, and MTC analyzed the data. APS, IJ, IP, and MTC wrote the paper.

\section{Acknowledgments}

We are grateful to S.C. Davis, T. Cao Wikramanayake, M. Boulina, C. Ricordi, and A. Barrientos for sharing laboratory resources and equipment. We also thank all our lab members for their continuous support and helpful criticisms. Our research is funded in part by the NIH grants AR060562, NR015649, and NR013881 (to MTC), Medline Research Grant Innovation Award granted by Wound Healing Foundation, Research Starter Grant granted by PhRMA Foundation (to IJ), SAC Award SAC-2013-19 (to MTC), SAC2016-9R1 (to RCS), and Dwoskin family gift to the Department of Dermatology and Cutaneous Surgery.

Address correspondence to: Marjana Tomic-Canic, Wound Healing and Regenerative Medicine Research Program, Dr. Phillip Frost Department of Dermatology and Cutaneous Surgery, University of Miami Miller School of Medicine, 1600 NW 10th Avenue, RMSB, Room 2023A, Miami, Florida 33136, USA. Phone: 305.243.7295; Email: mtcanic@med.miami.edu.

1. Eming SA, Martin P, Tomic-Canic M. Wound repair and regeneration: mechanisms, signaling, and translation. Sci Transl Med. 2014;6(265):265sr6

2. Sen CK, et al. Human skin wounds: a major and snowballing threat to public health and the economy. Wound Repair Regen. 2009;17(6):763-771.

3. Braun LR, Fisk WA, Lev-Tov H, Kirsner RS, Isseroff RR. Diabetic foot ulcer: an evidence-based treatment update. Am J Clin Dermatol. 2014;15(3):267-281.

4. Robbins JM, Strauss G, Aron D, Long J, Kuba J, Kaplan Y. Mortality rates and diabetic foot ulcers: is it time to communicate mortality risk to patients with diabetic foot ulceration? J Am Podiatr Med Assoc. 2008;98(6):489-493.

5. Brem H, Tomic-Canic M. Cellular and molecular basis of wound healing in diabetes. J Clin Invest. 2007;117(5):1219-1222.

6. Alavi A, et al. Diabetic foot ulcers: Part I. Pathophysiology and prevention. J Am Acad Dermatol. 2014;70(1):1.e1-1.e18

7. Asai J, et al. Topical simvastatin accelerates wound healing in diabetes by enhancing angiogenesis and lymphangiogenesis. Am $J$ Pathol. 2012;181(6):2217-2224.

8. Evangelista MT, Casintahan MF, Villafuerte LL. Simvastatin as a novel therapeutic agent for venous ulcers: a randomized, double-blind, placebo-controlled trial. Br J Dermatol. 2014;170(5):1151-1157.

9. Stojadinovic O, Lebrun E, Pastar I, Kirsner R, Davis SC, Tomic-Canic M. Statins as potential therapeutic agents for healing disorders. Expert Rev Dermatol. 2010;5(6):689-698.

10. Takwi AA, et al. A statin-regulated microRNA represses human c-Myc expression and function. EMBO Mol Med. 2012;4(9):896-909.

11. Sawaya AP, et al. Topical mevastatin promotes wound healing by inhibiting the transcription factor c-Myc via the glucocorticoid receptor and the long non-coding RNA Gas5. J Biol Chem. 2018;293(4):1439-1449.

12. Cervantes J, Jimenez JJ, DelCanto GM, Tosti A. Treatment of alopecia areata with Simvastatin/Ezetimibe. J Investig Dermatol Symp Proc. 2018;19(1):S25-S31.

13. Bu DX, et al. Statin-induced Krüppel-like factor 2 expression in human and mouse T cells reduces inflammatory and pathogenic responses. J Clin Invest. 2010;120(6):1961-1970.

14. Rosch JW, et al. Statins protect against fulminant pneumococcal infection and cytolysin toxicity in a mouse model of sickle cell disease. J Clin Invest. 2010;120(2):627-635.

15. Fox JD, et al. Statins may be associated with six-week diabetic foot ulcer healing. Wound Repair Regen. 2016;24(2):454-457.

16. Johansen OE, et al. Diabetic foot ulcer burden may be modified by high-dose atorvastatin: a 6-month randomized controlled pilot trial. J Diabetes. 2009;1(3):182-187.

17. Repertinger SK, Campagnaro E, Fuhrman J, El-Abaseri T, Yuspa SH, Hansen LA. EGFR enhances early healing after cutaneous incisional wounding. J Invest Dermatol. 2004;123(5):982-989.

18. Nobes CD, Hall A. Rho, rac, and cdc42 GTPases regulate the assembly of multimolecular focal complexes associated with actin stress fibers, lamellipodia, and filopodia. Cell. 1995;81(1):53-62.

19. Brem H, et al. Molecular markers in patients with chronic wounds to guide surgical debridement. Mol Med. 2007;13(1-2):30-39.

20. Jozic I, et al. Pharmacological and Genetic Inhibition of Caveolin-1 Promotes Epithelialization and Wound Closure. Mol Ther. 2019;27(11):1992-2004.

21. Hayer A, Stoeber M, Ritz D, Engel S, Meyer HH, Helenius A. Caveolin-1 is ubiquitinated and targeted to intralumenal vesicles 
in endolysosomes for degradation. J Cell Biol. 2010;191(3):615-629.

22. Darwin E, Tomic-Canic M. Healing chronic wounds: current challenges and potential solutions. Curr Dermatol Rep. 2018;7(4):296-302.

23. Pastar I, Wong LL, Egger AN, Tomic-Canic M. Descriptive vs mechanistic scientific approach to study wound healing and its inhibition: Is there a value of translational research involving human subjects? Exp Dermatol. 2018;27(5):551-562.

24. Sen CK. Human wounds and its burden: an updated compendium of estimates. Adv Wound Care (New Rochelle). 2019;8(2):39-48. 25. Pastar I, et al. Epithelialization in wound healing: a comprehensive review. Adv Wound Care (New Rochelle). 2014;3(7):445-464

26. Grande-García A, et al. Caveolin-1 regulates cell polarization and directional migration through Src kinase and Rho GTPases. $J$ Cell Biol. 2007;177(4):683-694.

27. Nethe M, et al. Focal-adhesion targeting links caveolin-1 to a Rac1-degradation pathway. J Cell Sci. 2010;123(Pt 11):1948-1958.

28. Jozic I, et al. Stress signals, mediated by membranous glucocorticoid receptor, activate PLC/PKC/GSK-3 $\beta / \beta$-catenin pathway to inhibit wound closure. J Invest Dermatol. 2017;137(5):1144-1154.

29. Slominski A, Wortsman J, Tuckey RC, Paus R. Differential expression of HPA axis homolog in the skin. Mol Cell Endocrinol. 2007;265-266:143-149.

30. Slominski AT, Manna PR, Tuckey RC. Cutaneous glucocorticosteroidogenesis: securing local homeostasis and the skin integrity. Exp Dermatol. 2014;23(6):369-374.

31. Vukelic S, et al. Cortisol synthesis in epidermis is induced by IL-1 and tissue injury. J Biol Chem. 2011;286(12):10265-10275

32. Bist A, Fielding PE, Fielding CJ. Two sterol regulatory element-like sequences mediate up-regulation of caveolin gene transcription in response to low density lipoprotein free cholesterol. Proc Natl Acad Sci U S A. 1997;94(20):10693-10698.

33. Fielding CJ, Bist A, Fielding PE. Caveolin mRNA levels are up-regulated by free cholesterol and down-regulated by oxysterols in fibroblast monolayers. Proc Natl Acad Sci U S A. 1997;94(8):3753-3758.

34. Couet J, Li S, Okamoto T, Ikezu T, Lisanti MP. Identification of peptide and protein ligands for the caveolin-scaffolding domain. Implications for the interaction of caveolin with caveolae-associated proteins. J Biol Chem. 1997;272(10):6525-6533

35. Okamoto T, Schlegel A, Scherer PE, Lisanti MP. Caveolins, a family of scaffolding proteins for organizing "preassembled signaling complexes" at the plasma membrane. J Biol Chem. 1998;273(10):5419-5422.

36. Fridolfsson HN, Roth DM, Insel PA, Patel HH. Regulation of intracellular signaling and function by caveolin. FASEB J. 2014;28(9):3823-3831

37. Patel HH, Murray F, Insel PA. Caveolae as organizers of pharmacologically relevant signal transduction molecules. Annu Rev Pharmacol Toxicol. 2008;48:359-391.

38. Evangelopoulos ME, Weis J, Krüttgen A. Mevastatin-induced neurite outgrowth of neuroblastoma cells via activation of EGFR. J Neurosci Res. 2009;87(9):2138-2144.

39. Pike LJ, Casey L. Cholesterol levels modulate EGF receptor-mediated signaling by altering receptor function and trafficking Biochemistry. 2002;41(32):10315-10322.

40. Barrientos S, Brem H, Stojadinovic O, Tomic-Canic M. Clinical application of growth factors and cytokines in wound healing Wound Repair Regen. 2014;22(5):569-578.

41. Pastar I, et al. Attenuation of the transforming growth factor beta-signaling pathway in chronic venous ulcers. Mol Med. 2010;16(3-4):92-101.

42. Falanga V, Eaglstein WH, Bucalo B, Katz MH, Harris B, Carson P. Topical use of human recombinant epidermal growth factor (h-EGF) in venous ulcers. J Dermatol Surg Oncol. 1992;18(7):604-606.

43. Randolph RK, Simon M. Characterization of retinol metabolism in cultured human epidermal keratinocytes. J Biol Chem. 1993;268(13):9198-9205.

44. Stojadinovic O, Tomic-Canic M. Human ex vivo wound healing model. Methods Mol Biol. 2013;1037:255-264. 